\title{
Optimum Complementary Control for Stable Systems of the Positive Linear Time Invariant
}

\author{
Kontrol Komplemeter Yang Optimal Untuk Sistem-Sistem Stabil dari \\ Invarian Waktu Linier Positif
}

\author{
Nurweni Putri ${ }^{1 *}$, Iswan Rina ${ }^{2^{*}}$
}

\begin{abstract}
The optimal control problem is defined as a problem in selecting a controller $\mathbf{u}(\mathrm{t})$ in a continuous linear system, so that it can provide the optimum value for a given objective function. The $\mathbf{u}(t)$ controller is expected to control the system so that it produces the desired output. In this research, it will be studied about how to select and construct the optimal controller $\mathbf{u}(\mathrm{t})$ in the Linear Time Invariant MIMO system positive stable, so that the given system will remain positive when given constant disturbance.
\end{abstract}

Keywords: Positive Stable MIMO System, Optimal Complementary Control, Hurwitz Matrix, Constant Disturbace.

\begin{abstract}
Abstrak
Masalah kontrol optimal didefinisikan sebagai masalah dalam pemilihan suatu pengontrol $\mathbf{u}(\mathrm{t})$ pada sistem linear kontinu, sedemikian sehingga dapat memberikan nilai optimum bagi fungsi objektif yang diberikan. Pengontrol $\mathbf{u}(\mathrm{t})$ ini diharapkan bisa mengontrol sistem sehingga menghasilkan output sesuai dengan yang diinginkan. Dalam penelitian ini akan dikaji tentang bagaimana memilih dan mengkonstruksi pengontrol optimal $\mathbf{u}(\mathrm{t})$ pada sistem MIMO Linear Time Invariant stabil positif, sehingga sistem yang diberikan akan tetap stabil positif saat diberikan disturbance konstan.
\end{abstract}

Kata kunci: Sistem MIMO stabil positif, Kontrol Komplementer Optimal, Matriks Hurwitz, Disturbace Konstan.

\section{PENDAHULUAN}

Masalah kontrol optimal didefinisikan sebagai suatu masalah memilih suatu pengontrol $\mathbf{u}(t)$ yang bergantung terhadap waktu $t$, sedemikian sehingga memberikan nilai optimum bagi fungsi objektif [13]. Perhatikan sistem LTI yang didefinisian pada [14] berikut

$$
\begin{aligned}
& \dot{\mathbf{x}}=A \mathbf{x}+B \mathbf{u} \\
& \mathbf{y}=C \mathbf{x}+D \mathbf{u}
\end{aligned}
$$

${ }^{1,2}$ Unversitas Dharma Andalas

Email: ${ }^{1}$ nurweni@unidha.ac.id' ; iswanrina0@gmail.com

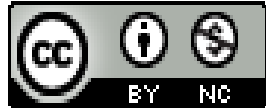

This work is licensed under a Creative Commons Attribution-NonCommercial 4.0 International License 


\section{Jurnal Matematika, Statistika \& Komputasi}

\section{Nurweni Putri, Iswan Rina}

dengan $\mathbf{x} \in \mathrm{R}^{n}$ menyatakan keadaan, $\mathbf{y} \in \mathrm{R}^{r}$ menyatakan output, $\mathbf{u} \in \mathrm{R}^{m}$ menyatakan kontrol (input), $A \in \mathrm{R}^{n \times n}, B \in \mathrm{R}^{n \times m}, C \in \mathrm{R}^{r \times n}$, dan $D \in \mathrm{R}^{r \times m}$. Kontrol komplementer optimal merupakan masalah penentuan suatu pengontrol optimal $\mathbf{u}(t) \in \mathrm{R}^{\mathrm{m}}$ yang memenuhi sistem (1) dan meminimumkan fungsi objektif

$$
J(\mathbf{u})=\int_{0}^{\infty}\left(\mathbf{x}^{T} Q \mathbf{x}+\mathbf{u}^{T} \mathbf{u}\right) d t
$$

dimana $Q \in \mathrm{R}^{n \times n}$ adalah matriks simetris [16].

Berdasarkan [9], sistem (1) dikatakan positif jika untuk setiap keadaan awal nonnegatif dan input nonnegatif. Sehingga keadaan dan output pada waktu $t$ adalah nonnegatif, yaitu $\mathbf{x}(t) \in \mathrm{R}_{+}^{n}$ dan $\mathbf{y}(t) \in \mathrm{R}_{+}^{r}, \forall t \in \mathrm{R}_{+}$. Selain itu berdasarkan [15], suatu syarat cukup agar sistem (1) stabil adalah $A$ matriks Hurwitz, yaitu bagian riil dari semua nilai eigen dari $A$ adalah negatif atau $\operatorname{Re}(\lambda)<0, \forall \lambda$. Dalam [4] dan [6], dinyatakan juga bahwa sistem (1) adalah positif jika dan hanya jika $A$ adalah matriks Metzler dan matriks-matriks $B$, $C$, dan $D$ adalah matriks nonnegatif. Dengan demikian, agar sistem (1) stabil positif maka $A$ haruslah matriks Hurwitz Metzler.

Seiring dengan perkembangan dalam berbagai bidang, masalah kontrol optimal telah mengalami berbagai modifikasi. Pada penelitian ini adanya penambahan faktor gangguang (disturbance) pada sistem (1), sebagai berikut :

$$
\begin{aligned}
& \dot{\mathbf{x}}=A \mathbf{x}+B \mathbf{u}+E \varphi \\
& \mathbf{y}=C \mathbf{x}+D \mathbf{u}+F \varphi
\end{aligned}
$$

dimana $E \in \mathrm{R}^{n \times q}, F \in \mathrm{R}^{r \times q}$ dan $\varphi$ menyatakan vektor gangguan (disturbance).

Pendefinisian kontrol komplementer optimal untuk sistem (3) diperluas, yaitu: menentukan kontrol komplementer optimal $\mathbf{u}(t) \in \mathrm{R}^{m}$ yang memenuhi sistem (3) dan meminimumkan fungsi objektif berikut:

$$
J(\mathbf{u})=\int_{0}^{\infty}\left(\boldsymbol{\varepsilon}^{T} Q \boldsymbol{\varepsilon}+\dot{\mathbf{u}}^{T} \dot{\mathbf{u}}\right) d t,
$$

dimana $Q \in \mathrm{R}^{n \times n}$ adalah matriks simetris.

Dalam prakteknya, output yang diperoleh dari sistem tidak selalu sesuai dengan yang diinginkan. Hali ini dipengaruhi oleh beberapa faktor, salah satunya muncul error pada sistem. Jika $\mathbf{y}_{\text {ref }} \in \mathrm{R}^{r}$ menyatakan vektor output yang diinginkan dan $\boldsymbol{\varepsilon}$ menyatakan error pada sistem, maka $\boldsymbol{\varepsilon}=\mathbf{y}-\mathbf{y}_{\text {ref }}$. Agar memperoleh $\mathbf{y}_{\text {ref }}$ yang maksimal, maka diperlukan suatu pengontrol u sedemikian sehingga $\boldsymbol{\varepsilon} \rightarrow 0$ bila $\mathrm{t} \rightarrow \infty$. Hubungan ini dapat dilihat dalam bentuk diagram berikut 


\section{Jurnal Matematika, Statistika \& Komputasi \\ Nurweni Putri, Iswan Rina}

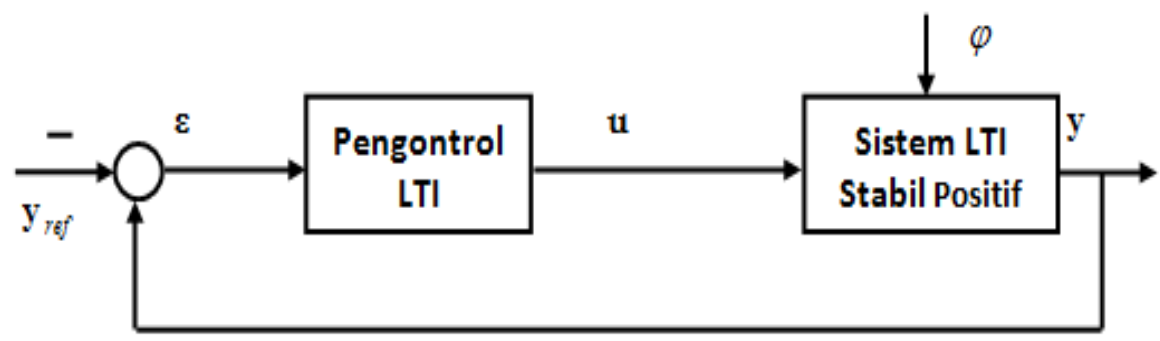

Gambar 1. Closed-Loop LTI System

Sehingga pada penelitian ini akan mengkaji bagaimana mengkonstruksi pengontrol optimal $\mathbf{u}(\mathrm{t})$ yang memenuhi sistem (3) dan meminimumkan fungsi objektif (4) sedemikian sehingga sistem tetap stabil positif saat diberikan gangguan (disturbance) konstan. Bersadarkan definisi dan syarat cukup dari sistem stabil positif, maka pengontrol optimal $\mathbf{u}(\mathrm{t})$ harus memenuhi kondisi berikut, yaitu:

1. Matriks $A+B \mathrm{~K}_{\mathrm{x}}$ adalah matriks Hurwitz,

2. Keadaan $\mathbf{x}(\mathrm{t})$ dan output $\mathbf{y}(\mathrm{t})$ adalah nonnegatif, yaitu, $\mathbf{x}(t) \in \mathrm{R}_{+}^{n}$ dan $\mathbf{y}(t) \in \mathrm{R}_{+}^{r}, \forall t \in \mathrm{R}_{+}$

3. $\boldsymbol{\varepsilon}=\mathbf{y}-\mathbf{y}_{\text {ref }} \rightarrow \mathbf{0}$ bila $\mathrm{t} \rightarrow \infty$.

\section{LANDASAN TEORI}

Beberapa teori dasar dan tinjauan hasil-hasil penelitian terdahulu yang terkait dengan topik bahasan pada penelitian ini.

Definisi 1 [8] Suatu matriks simetris $A \in \mathrm{R}^{n \times n}$ dikatakan definit positif jika $\forall \mathbf{x} \in \mathrm{R}^{n}, \mathbf{x} \neq \mathbf{0}$, berlaku $\mathbf{x}^{T} A \mathbf{x}>0$. Selain itu, $A$ dikatakan definit negatif jika $\forall \mathbf{x} \in \mathrm{R}^{n}, \mathbf{x} \neq \mathbf{0}$, berlaku $\mathbf{x}^{T} A \mathbf{x}<0$. Matriks A dikatakan nonnegatif, jika $a_{i j} \geq 0, \forall i, j$ dan $a_{i j}>0$ untuk sekurang-kurangnya satu entri.

Definisi 2 [7] Matriks $A=\left[a_{i j}\right] \in \mathrm{R}^{n \times n}$ dikatakan matriks Metzler jika entri-entri selain entri diagonal utamanya adalah nonnegatif, yaitu $a_{i j} \geq 0$ untuk $i \neq j, i, j=1,2, \ldots, n$.

Teorema 3 [4] Suatu matriks $A \in \mathrm{R}^{n \times n}$ adalah matriks Metzler jika dan hanya jika $e^{A t} \in \mathrm{R}_{+}^{n \times n}, \forall t \geq 0$.

Teorema 4 [15] Jika $A$ adalah matriks Metzler-Hurwitz, maka $-A^{-1}$ ada dan $-A^{-1} \in R_{+}^{n \times n}$.

Teorema 5 [7] Misalkan ada suatu matriks simetris definit positif $P$ yang memenuhi persamaan aljabar Riccati $A^{T} P+P A+Q-(P B)(P B)^{T}=O$ dan bagian riil dari semua nilai eigen matriks $A-B(B T)$ adalah negatif, maka

$$
\mathbf{u}^{*}(t)=K \mathbf{x}^{*}(t), t \geq 0
$$

dimana $K=-(P B) T$, dengan $\mathbf{x}^{*}(t)$ adalah solusi dari persamaan diferensial

$$
\dot{\mathbf{x}}^{*}(t)=\left(A-B(P B)^{T}\right) \mathbf{x}^{*}(t)
$$




\section{Jurnal Matematika, Statistika \& Komputasi \\ Nurweni Putri, Iswan Rina}

meminimumkan $J(\mathbf{u})$, dan $J\left(\mathbf{u}^{*}\right)=\mathbf{x}^{T}(0) P \mathbf{x}(0)$.

Selanjutnya, notasikan pengontrol dalam keadaan steady-state sebagai $\mathbf{u}_{\mathrm{s}}$ dan pengontrol dalam keadaan optimal sebagai $\mathbf{u}$. Teorema berikut merupakan syarat perlu untuk eksistensi dari $\mathbf{u}(\mathrm{t})$.

Teorema 6 [18] Misalkan rank $\left(D-C A^{-1} B\right)=r$ dan $K_{r}, K_{d}$ didefinisikan sebagai berikut

$$
\begin{aligned}
& K_{r}=\left(D-C A^{-1} B\right)^{-1} \\
& K_{d}=-K_{r}\left(F-C A^{-1} E\right)^{-1}
\end{aligned}
$$

Jika $\mathbf{u}(\mathrm{t})$ ada, maka untuk sebarang $\mathbf{y}_{\text {ref }}$ dan sebarang $\varphi$, $\mathbf{u}_{\mathrm{s}}$ memenuhi persamaan berikut

$$
\mathbf{u}_{\mathrm{s}}=K_{r} \mathbf{y}_{\text {ref }}+K_{d} \varphi
$$

Bukti : Perhatikan bahwa dalam kondisi steady state berlaku

$$
\begin{aligned}
& \dot{\mathbf{x}}=\mathrm{A} \mathbf{x}_{\mathrm{s}}+\mathrm{B} \mathbf{u}_{\mathrm{s}}+\mathrm{E} \varphi=\mathbf{0} \\
& \mathbf{e}=\mathrm{C} \mathbf{x}_{\mathrm{s}}+\mathrm{D} \mathbf{u}_{\mathrm{s}}+\mathrm{F} \varphi-\mathbf{y}_{\mathrm{ref}}=\mathbf{0},
\end{aligned}
$$

dengan $\mathbf{x}_{\mathbf{s}}$ adalah keadaan steady state. Berdasarkan Teorema 4, maka persaman (8) dapat ditulis sebagai berikut

$$
\mathbf{x}_{\mathrm{s}}=-\mathrm{A}^{-1} \mathrm{~B} \mathbf{u}_{\mathrm{s}}-\mathrm{A}^{-1} \mathrm{E} \varphi
$$

dengan mensubtitusikan bentuk diatas kedalam persamaan (9), diperoleh

$$
\begin{aligned}
\mathbf{0} & =C\left[-A^{-1} B \mathbf{u}_{\mathrm{s}}-A^{-1} E \varphi\right]+D \mathbf{u}_{\mathrm{s}}+F \varphi-\mathbf{y}_{r e f} \\
\mathbf{u}_{\mathrm{s}} & =\left[D-C A^{-1} B\right] \mathbf{y}_{r e f}-\left[D-C A^{-1} B\right]^{-1}\left[F-C A^{-1} E\right] \varphi \\
\mathbf{u}_{\mathrm{s}} & =K_{r} \mathbf{y}_{r e f}+K_{d} \varphi
\end{aligned}
$$

\section{METODE PENELITIAN}

Berikut adalah langkah-langkah yang akan dilakukan dalam menyelesaikan penelitian ini :

1. Melakukan studi literatur dengan mengumpulkan referensi yang relevan berupa buku teks, jurnal, dan artikel terkait di internet.

2. Mengumpulkan berbagai defenisi dan teorema yang berkaitan dengan penelitian.

3. Mengkonstruksi kontrol optimal sehingga tiga konsdisi terpenuhi.

4. Menguji bentuk kontrol optimal yang telah dikonstruksi pada contoh soal dengan menggunakan software MATLAB R201. Apakah memenuhi ketiga kondisi atau tidak. Jika memenuhi, maka sistem stabil positif.

5. Melakukan interpretasi terhadap hasil-hasil yang diperoleh.

\section{HASIL DAN PEMBAHASAN}

Berikut akan diberikan langkah untuk mengkonstruksi $\mathbf{u}(\mathrm{t})$ agar sistem tetap stabil positif saat diberikan gangguan konstan. Definisikan variabel baru $\mu$, dimana $\dot{\mu}=\eta \boldsymbol{\varepsilon}$, maka sistem (3) dengan $\boldsymbol{\varepsilon}=\mathbf{y}-\mathbf{y}_{\text {ref }}$ dapat ditulis sebagai berikut 


\section{Jurnal Matematika, Statistika \& Komputasi}

\section{Nurweni Putri, Iswan Rina}

$$
\left[\begin{array}{c}
\dot{\mathbf{x}} \\
\varepsilon
\end{array}\right]=\left[\begin{array}{ll}
A & O \\
C & O
\end{array}\right]\left[\begin{array}{l}
\mathbf{x} \\
\mu
\end{array}\right]+\left[\begin{array}{l}
B \\
D
\end{array}\right] \mathbf{u}+\left[\begin{array}{l}
\mathrm{E} \\
\mathrm{F}
\end{array}\right] \varphi-\left[\begin{array}{l}
O \\
I_{r}
\end{array}\right] \mathbf{y}_{\text {ref }}
$$

Perhatikan bahwa disturbance $\varphi$ dan output yang diinginkan $\mathbf{y}_{\text {ref }}$ adalah konstan, maka bentuk diatas dapat diubah menjadi

$$
\left[\begin{array}{c}
\ddot{\mathbf{x}} \\
\dot{\boldsymbol{\varepsilon}}
\end{array}\right]=\left[\begin{array}{cc}
A & O \\
C & O
\end{array}\right]\left[\begin{array}{c}
\dot{\mathbf{x}} \\
\eta \mathbf{\varepsilon}
\end{array}\right]+\left[\begin{array}{c}
B \\
D
\end{array}\right] \dot{\mathbf{u}}
$$

Oleh karena sistem (3) adalah stabil, maka kontrol $\dot{\mathbf{u}}$ yang akan ditemukan harus memenuhi sistem (11) dan meminimumkan

$$
J(\mathbf{u})=\int_{0}^{\infty}\left(\boldsymbol{\varepsilon}^{T} Q \boldsymbol{\varepsilon}+\dot{\mathbf{u}}^{T} \dot{\mathbf{u}}\right) d t
$$

dengan $Q=K_{r}{ }^{T} K_{r}$ dan $K_{r}$ didefinisikan seperti pada persamaan (5). Sistem (11) dan (12) dapat ditulis kembali dalam bentuk masalah optimasi baru, sebagai berikut:

$$
\begin{aligned}
\min _{\mathbf{u}} J & =\int_{0}^{\infty}\left[\hat{\mathbf{x}}^{T}(t) \hat{Q} \hat{\mathbf{x}}(t)+\hat{\mathbf{u}}^{T}(t) \hat{\mathbf{u}}(t)\right] \mathrm{dt} \\
\text { s.t } \dot{\hat{\mathbf{x}}} & =\hat{\mathrm{A}} \hat{\mathbf{x}}+\hat{B} \hat{\mathbf{u}},
\end{aligned}
$$

dimana

$$
\dot{\hat{\mathbf{x}}}=\left[\begin{array}{c}
\ddot{\mathbf{x}} \\
\dot{\boldsymbol{\varepsilon}}
\end{array}\right], \quad \hat{\mathbf{x}}=\left[\begin{array}{c}
\dot{\mathbf{x}} \\
\boldsymbol{\varepsilon}
\end{array}\right], \quad \hat{\mathrm{A}}=\left[\begin{array}{cc}
A & O \\
C & O
\end{array}\right], \quad \hat{B}=\left[\begin{array}{c}
B \\
O
\end{array}\right], \quad \hat{\mathrm{Q}}=\left[\begin{array}{cc}
O & O \\
O & Q
\end{array}\right], \quad \hat{\mathbf{u}}=\dot{\mathrm{u}}
$$

Berdasarkan teori dari masalah kontrol komplementer optimal, bentuk kontrol optimal dari (13) adalah

$$
\hat{\mathbf{u}}^{*}=-(P \hat{B})^{\mathrm{T}} \hat{\mathbf{x}}^{*}
$$

dimana $\mathrm{P}$ adalah matriks simetris definit positif yang merupakan solusi dari persamaan aljabar Riccati dan $\hat{\mathbf{x}}^{*}$ adalah keadaan optimal yang terkait dengan kontrol komplementer optimal $\hat{\mathbf{u}}^{*}$ dari persamaan (13). Perhatikan bahwa $K_{\xi}=-(P \hat{B})^{T}$, maka bentuk kontrol (14) dapat diubah menjadi

$$
\hat{\mathbf{u}}^{*}=K_{\xi}\left[\begin{array}{l}
\mathbf{x}^{*} \\
\mu
\end{array}\right]
$$

Tulis matriks $K_{\xi}$ sebagai berikut

$$
K_{\xi}=\left[K_{\mathbf{x}}(\xi) \quad K_{\boldsymbol{\eta}}(\xi)\right]=\left[\xi \bar{K}_{\mathbf{x}}(\xi) \quad \xi \bar{K}_{\mathbf{\eta}}(\xi)\right]
$$

dimana $\xi>0$. Akibatnya diperoleh 


\section{Jurnal Matematika, Statistika \& Komputasi}

\section{Nurweni Putri, Iswan Rina}

$$
\begin{aligned}
K_{\xi} & =-\hat{B}^{T} P \\
& =-\left[\begin{array}{ll}
B^{T} & D^{T}
\end{array}\right]\left[\begin{array}{ll}
P_{11} & P_{12} \\
P_{21} & P_{22}
\end{array}\right] \\
& =\left[-\left(B^{T} P_{11}+D^{T} P_{21}\right)-\left(B^{T} P_{12}+D^{T} P_{22}\right)\right]
\end{aligned}
$$

Dari persamaan aljabar Riccati pada diperoleh

$$
\begin{aligned}
\left(P_{21} B+P_{22} D\right)\left(B^{T} P_{12}+D^{T} P_{22}\right) & =\xi^{2} Q \\
K_{r}^{T} K_{r} & =\left(-\xi K_{\eta}^{T}\right)\left(-\xi K_{\eta}\right)
\end{aligned}
$$

ini mengakibatkan $K_{\eta}=-\xi K_{r}$, sehingga

$$
K_{\xi}=\left[\xi \bar{K}_{\mathbf{x}}(\xi)-\xi K_{r}(\xi)\right]
$$

Selanjutnya dengan mensubtitusikan (3) kedalam (15) maka didapatkan bentuk persamaan sebagai berikut

$$
\left[\begin{array}{c}
\dot{\mathbf{x}}^{*} \\
\dot{\mathbf{u}}^{*}
\end{array}\right]=\left[\begin{array}{cc}
A & B \\
K_{\mathbf{x}} \mathrm{A}+K_{\eta} C & K_{\mathbf{x}} \mathrm{B}+K_{\eta} D
\end{array}\right]\left[\begin{array}{l}
\mathbf{x}^{*} \\
\mathbf{u}^{*}
\end{array}\right]+\left[\begin{array}{c}
\mathrm{E} \\
K_{\mathbf{x}} \mathrm{E}+K_{\eta} \mathrm{F}
\end{array}\right] \varphi-\left[\begin{array}{c}
O \\
K_{\eta}
\end{array}\right] \mathbf{y}_{\text {ref }} .
$$

Misalkan pada kondisi steady-state, $\mathbf{x}_{\mathbf{s}}$ dan $\mathbf{u}_{\mathbf{s}}$ tidak bergantung kepada $\xi$, maka dapat dilakukan transformasi berikut

$$
\begin{aligned}
& \mathbf{z}=\mathbf{x}^{*}-\mathbf{x} \\
& \mathbf{q}=\mathbf{u}^{*}-\mathbf{u}_{\mathrm{s}}
\end{aligned}
$$

sehingga diperoleh

$$
\left[\begin{array}{c}
\dot{\mathbf{z}} \\
\dot{\mathbf{q}}
\end{array}\right]=\left[\begin{array}{cc}
A & B \\
K_{\mathbf{x}} \mathrm{A}+K_{\eta} C & K_{\mathbf{x}} \mathrm{B}+K_{\eta} D
\end{array}\right]\left[\begin{array}{l}
\mathbf{z} \\
\mathbf{q}
\end{array}\right]=\left[\begin{array}{cc}
A & B \\
\xi \bar{K}_{\mathbf{x}} \mathrm{A}-\xi K_{r} C & \xi \bar{K}_{\mathbf{x}} \mathrm{B}-\xi K_{r} D
\end{array}\right]\left[\begin{array}{l}
\mathbf{z} \\
\mathbf{q}
\end{array}\right]
$$

Definisikan $\xi d t=d \tau$, maka transformasi sistem menjadi

$$
\left[\begin{array}{c}
\oplus \\
\xi \mathbf{z} \\
\xi \stackrel{\oplus}{\mathbf{q}}
\end{array}\right]=\left[\begin{array}{cc}
A & B \\
\bar{K}_{\mathbf{x}} \mathrm{A}-K_{r} C & \bar{K}_{\mathbf{x}} \mathrm{B}-K_{r} D
\end{array}\right]\left[\begin{array}{l}
\mathbf{z} \\
\mathbf{q}
\end{array}\right],
$$

$\operatorname{dimana} \xi_{\mathbf{z}}^{\stackrel{\oplus}{\mathbf{z}}}=\stackrel{\oplus}{\mathbf{z}}$ dan $\xi_{\mathbf{q}} \mathbf{\oplus}=\stackrel{\oplus}{\mathbf{q}}$. Jika $\xi \rightarrow 0$, maka diperoleh

$$
\mathbf{z}=-A^{-1} B \mathbf{q},
$$

akibatnya 


\section{Jurnal Matematika, Statistika \& Komputasi}

\section{Nurweni Putri, Iswan Rina}

$$
\begin{aligned}
\stackrel{\oplus}{\mathbf{q}} & =\left(\bar{K}_{\mathbf{x}} \mathrm{A}-K_{r} C\right)\left(-A^{-1} B\right) \mathbf{q}+\left(\bar{K}_{\mathbf{x}} \mathrm{B}-K_{r} D\right) \mathbf{q} \\
& =-\bar{K}_{\mathbf{x}} B \mathbf{q}+K_{r} C A^{-1} B \mathbf{q}+\bar{K}_{\mathbf{x}} \mathrm{B} \mathbf{q}-K_{r} D \mathbf{q} \\
& =K_{r}\left(-D+C A^{-1} B\right) \mathbf{q} \\
& =\left(D-C A^{-1} B\right)^{-1}-\left(D-C A^{-1} B\right) \mathbf{q} \\
& =-\mathbf{q},
\end{aligned}
$$

dengan solusi

$$
\mathbf{q}(\tau)=\boldsymbol{\varepsilon}^{-\tau} \mathbf{q}(0),
$$

dimana $\xi t=\tau$ dan $\mathbf{q}(0)=\mathbf{u}^{*}(0)-\mathbf{u}_{\mathrm{s}}$. Subtitusikan (20) ke persamaan (19) diperoleh

$$
\begin{aligned}
\mathbf{u}^{*} & =\mathbf{q}+\mathbf{u}_{s} \\
& =\boldsymbol{\varepsilon}^{-\xi_{t}}\left(\mathbf{u}(0)-\mathbf{u}_{\mathrm{s}}\right)+\mathbf{u}_{\mathrm{s}} .
\end{aligned}
$$

Perhatikan persamaan (21), jika $\mathrm{t} \rightarrow \infty$ maka $\mathbf{u}^{*} \rightarrow \mathbf{u}_{\mathrm{s}}$. Dengan kata lain, jika $\mathrm{t} \rightarrow \infty$ maka $\boldsymbol{\varepsilon} \rightarrow 0$ akibatnya kondisi yang ketiga terpenuhi.

Selanjutnya, kondisi yang kedua sudah terjamin dari sifat kenonnegatifan $\mathbf{u}^{*}$, yaitu keadaan $\mathbf{x}^{*}$ dan output $\mathbf{y}$ adalah nonnegatif untuk setiap $\mathrm{t} \geq 0$. Untuk menunjukan kondisi yang pertama, maka subtitusikan bentuk dari $\mathbf{u}^{*}$ ke dalam sistem (3), sehingga diperoleh

$$
\begin{aligned}
\dot{\mathbf{x}}^{*} & =\mathrm{A} \mathbf{x}^{*}+\mathrm{Bu}^{*}+\mathrm{E} \varphi \\
& =\mathrm{A} \mathbf{x}^{*}+\mathrm{B}\left[\begin{array}{ll}
\mathrm{K}_{\mathbf{x}} & K_{\eta}
\end{array}\right]\left[\begin{array}{c}
\mathbf{x}^{*} \\
\eta
\end{array}\right]+\mathrm{E} \varphi \\
& =\left(A+B \mathrm{~K}_{\mathbf{x}}\right) \mathbf{x}^{*}+B K_{\eta} \eta+\mathrm{E} \varphi
\end{aligned}
$$

Perhatikan bahwa A adalah matriks Metzler Hurwitz, sehingga sistem (22) stabil positif. Akibatnya terdapat $\mathrm{K}_{\mathrm{x}} \in \mathrm{R}^{\mathrm{m} \times \mathrm{n}}$ sedemikian sehingga $A+B \mathrm{~K}_{\mathrm{x}}$ adalah matriks Metzler Hurwitz. Dengan terpenuhinya ketiga kondisi oleh pengontrol $\mathbf{u}^{*}$ yang memenuhi sistem (3) dan meminimumkan (4), maka kontrol optimal agar sistem tetap postif stabil ketika diberikan gangguan konstan adalah

$$
\mathbf{u}^{*}=\left[\begin{array}{ll}
\mathrm{K}_{\mathbf{x}} & K_{\eta}
\end{array}\right]\left[\begin{array}{c}
\mathbf{x}^{*} \\
\eta
\end{array}\right] .
$$

Contoh 1. Diberikan sistem MIMO LTI stabil positif sebagai berikut 


\section{Jurnal Matematika, Statistika E Komputasi}

\section{Nurweni Putri, Iswan Rina}

$$
\begin{aligned}
& \dot{\mathbf{x}}=\left[\begin{array}{ccc}
-0.8 & 0 & 0 \\
0 & -0.7 & 0 \\
0.8 & 0.7 & -0.5
\end{array}\right] \mathbf{x}+\left[\begin{array}{cc}
0.5 & 0 \\
0.5 & 0 \\
0 & 1
\end{array}\right] \mathbf{u}+\left[\begin{array}{c}
0.5 \\
0.5 \\
0
\end{array}\right] \varphi \\
& \mathbf{y}=\left[\begin{array}{lll}
0 & 1 & 0 \\
1 & 0 & 1
\end{array}\right] \mathbf{x}+\left[\begin{array}{cc}
0.5 & 0 \\
0 & 1
\end{array}\right] \mathbf{u}+\left[\begin{array}{c}
0.5 \\
0
\end{array}\right] \varphi
\end{aligned}
$$

Pada contoh ini diasumsikan nilai awal $\mathbf{x}(0)=\left[\begin{array}{lll}1.2 & 0.4 & 1.8\end{array}\right]^{T}, \quad \varphi=0.1$ dan output yang diinginkan $\mathbf{y}_{\text {ref }}=\left[\begin{array}{ll}0.1 & 0.9\end{array}\right]^{T}$. Hitung nilai $\mathbf{u}$ dan $\mathbf{x}$ dalam keadaan steady-state maka diperoleh :

$$
\mathbf{u}_{\mathrm{s}}=\left[\begin{array}{l}
0.05 \\
0.22
\end{array}\right], \quad \mathbf{x}_{s}=\left[\begin{array}{l}
0.0938 \\
0.1071 \\
0.8000
\end{array}\right] .
$$

Selanjutnya hitung nilai dari $A+B \mathrm{~K}_{\mathrm{x}}$ dan nilai eigennya, yaitu:

$$
A+B \mathrm{~K}_{\mathrm{x}}=\left[\begin{array}{ccc}
0.7362 & 0.0195 & 0.0417 \\
0.0638 & -0.6805 & 0.0417 \\
1.1029 & 0.7440 & -0.2867
\end{array}\right]
$$

dengan nilai eigen adalah $-0.1422,-0.8294$, dan -0.7318 . Berdasarkan Teorema 4 , matriks $A+B \mathrm{~K}_{\mathrm{x}}$ adalah Metzler-Hurwitz, sehingga sifat (1) terpenuhi.

Perhatikan plot dari keadaan $\mathbf{x}(t)$ dan output $\mathbf{y}(t)$ berturut-turut diberikan pada gambar berikut :
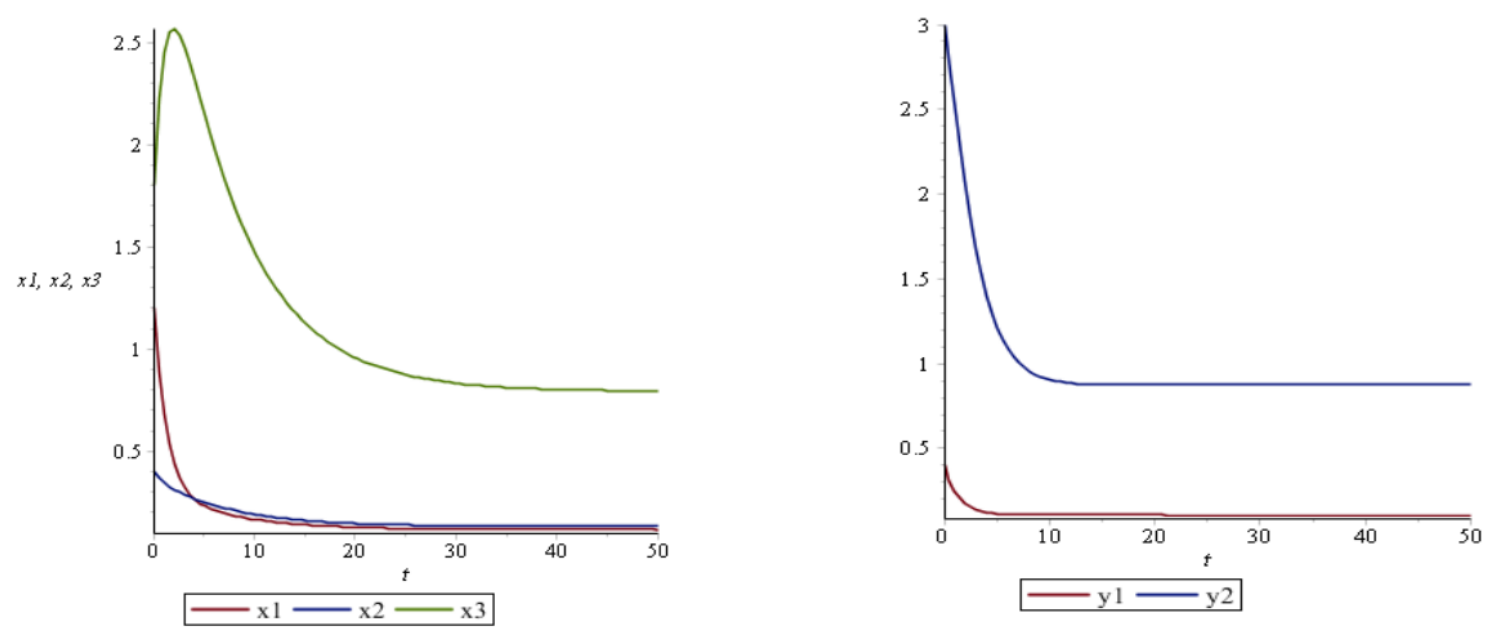

Gambar 2. Plot fungsi $\mathbf{x}(\mathrm{t})$ dan $\mathbf{y}(\mathrm{t})$ terhadap $\mathrm{t}$ 


\section{Jurnal Matematika, Statistika E Komputasi \\ Nurweni Putri, Iswan Rina}

Dari grafik yang diperoleh pada Gambar 2 diketahui bahwa keadaan $\mathbf{x}(\mathrm{t})$ dan output $\mathbf{y}(\mathrm{t})$ adalah nonnegatif, sehingga kondisi (2) terpenuhi. Adapun plot dari kontrol optimal $\mathbf{u}(\mathrm{t})$ diperoleh sebagai berikut :

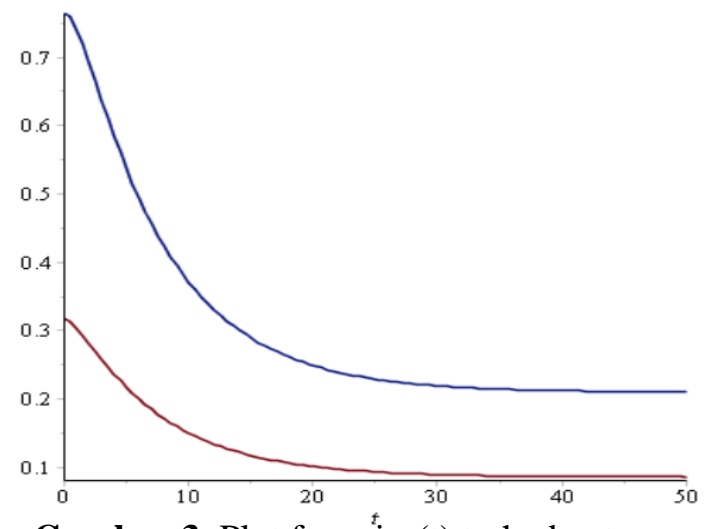

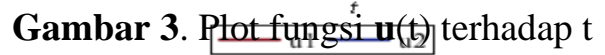

Dari grafik $\mathbf{u}(\mathrm{t})$ pada Gambar 3 dapat dilihat bahwa jika $\mathrm{t} \rightarrow \infty$ maka $\mathbf{u}^{*} \rightarrow \mathbf{u}_{\mathrm{s}}$. Dengan kata lain, jika $\mathrm{t}$ $\rightarrow \infty$ maka $\boldsymbol{\varepsilon} \rightarrow 0$ akibatnya kondisi (3) terpenuhi. Karena semua sifat terpenuhi, maka sistem adalah stabil positif.

\section{KESIMPULAN}

Bentuk kontrol optimal yang memenuhi sistem (3) dan meminimumkan (4), sedemikian sehingga sistem tetap stabil positif saat diberikan disturbance konstan adalah sebagai berikut

dengan $K_{\xi}=\left[K_{\mathbf{x}}(\xi) K_{n}(\xi)\right]=\left[\xi \bar{K}_{\mathbf{x}}(\xi)-\xi K_{r}(\xi)\right]$.

$$
\mathbf{u}(t)=K_{\xi}\left[\begin{array}{l}
\mathbf{x}^{*} \\
\eta
\end{array}\right],
$$

\section{DAFTAR PUSTAKA}

[1] Ariyanti Gregoria. 2020. Necessary and Sufficient Conditions for The Solutions of Linear Equations System. Jurnal Matematika, stattistika dan Komputasi, Vol.17 No.1, 82-88.

[2] Arif D., Adzkiya D., Apriliani E., \& Khasanah I. 2017. Model Reduction of Non-minimal DiscreteTime Linear-Time-Invariant Systems. MJMS

[3] Beauthier, C. dan Joseph J. Winkin. 2010. LQ-Optimal Control of Positive Linear System. Wiley Online Library.

[4] Farina, L. dan Rinaldi, S. 2000. Positive Linear Systems : Theory and Aplications. Wiley. New York.

[5] Firman. 2011. Modifikasi Kontro untuk Sistem Tak Linier Input Tunggal-Output Tunggal. Jurnal Matematika, stattistika dan Komputasi, Vol.7 No.2, 118-123.

[6] Guo Zongyi, David H., Jianguo G., Zheng W., Jarome \& Jing C. 2020. Metzler Matrix-Based Switching Control Scheme For Linear Systems With Prescribed Performance Guarantees. IFACPapersOnLine 53-2 (2020) 6428-6433.

[7] Hendricks. Elbert, Ole. Jannerup dan P. H. Sorensen. 2008. Linear Systems Control. Springer- Verlag Berlin Heidelberg.

[8] Kaczorek, T. 2001. Positive 1D and 2D Systems Metzler Matrices. SpringerVerlag Berlin Heidelberg. 


\section{Jurnal Matematika, Statistika E Komputasi}

\section{Nurweni Putri, Iswan Rina}

[9] Krokavec D. \& Filasova A. 2020. Reduced-Order Observers for Linear Metzlerian Systems. IFACPapersOnLine 53-2 (2020) 4553-6558.

[10] Kundu A., Daafouz J., \& Heemels. 2017. Stabilization of Discrete-Time Switched Linear Systems: Lyapunov-Metzler Inequalities versus S-procedure characterizations. IFAC-PapersOnLine 50-1 (2017) 3412-3417.

[11] Leenheer, P. dan D. Aeyels. 2001. Stabilization of Positive Linear Systems. Systems and Control Letters. 44: 259-271.

[12] Meyer. Carl D. 2000. Matrix Analysis and Applied Linear Algebra. SIAM

[13] Mitkowski. W. 2008. Dynamical Properties of Metzler Systems. Bulletin of The Polish Academy of Sciences Technical Sciences. Vol. 56, No. 4.

[14] Naidu, D.S. 2002. Optimal Control Systems. CRC Press, Idaho.

[15] Rama K. Yedavalli. 2018. Conditions for Hurwitz Stability / Instability of a Real matrix via its Sign Pattern with a Necessary and Sufficent Condition for Magnitude Independent Stability. IFACPapersOnLine 51-1 (2018) 663-667.

[16] Richard, Charles Jhonson. 1982. Inverse M-Matrices. Algebra Linear and Its Applications 47:195216.

[17] Roszak, B. dan Davidson, E. J. 2009. Necessary and Sufficient Conditions for Stabilizability of Positive LTI System. System and Control Letters 58(2009). 474-481.

[18] Roszak, B. dan Davidson, E. J. 2010. The Multivariable Servomechanism controller for SISO positive LTI System. IEEE Transactions on Automatic Control, 55(9), 2204-2209. 